\title{
Calculation of flexural capacity and rigidity for RC floor strengthened with bilateral steel-slab-bonded
}

\author{
$\mathrm{Ge} \mathrm{Wu}{ }^{1, a}$, Yanyang Zhao ${ }^{1, b}$ and Meng Guo ${ }^{2, \mathrm{c}}$ \\ ${ }^{1}$ Jilin Jianzhu University, Changchun, China \\ ${ }^{2}$ Beijing Institute of Architectural Design, Beijing,China \\ a454862380@qq.com, ${ }^{b} 593901830 @ q q . c o m,{ }^{c} 910613907 @ q q . c o m$
}

\begin{abstract}
Keywords:Bilateral steel-slab-bonded; Strengthened; RC floor; Flexural capacity; Rigidity; Detail measure

Abstract:Based on interrelated experiments research of $\mathrm{RC}$ beam strengthened with unilateral steel-slab-bonded and practical engineering needs, the technology of RC floor strengthened with bilateral steel-slab-bonded is presented. According to section internal force equation relation and classical theory about bended component rigidity, and considering of $\mathrm{RC}$ floor which is not uninstalled completely during strengthened process, the formula of flexural capacity and section rigidity about FC floor strengthened with bilateral steel-slab-bonded are established. Two type of detail measures are given for improving the bonded effect between slab and RC floor.
\end{abstract}

\section{Introduction}

The method using pasting steel plate reinforced to improve the bearing capacity of concrete floor, has been widely used in the buildings consolidation and reconstruction." Code for Design of Concrete Structures Reinforcement" is giving the corresponding design method and calculation formula and structural requirements ${ }^{[1]}$.the reinforcement methods of sticking steel are pasting unilateral steel-slab-bonded in the bottom of slab and the surface of slab in the joints. Steel plate mainly used to bear the pull force caused by the load change. The concrete compressive zone still rely on own compressive ability to resist the increase of the bending moment caused by external load. Under the condition of the floor thinner, although the bearing capacity of floor which is reinforced by unilateral steel-slab-bonded meet requirements, but the floor slab deflection and crack increase, which will bring a certain security risk. Many the precision instruments within factories and other devices is relatively sensitive to deformation and vibration of floor, too big deflection or vibration will influence the equipment's normal work and service life.

Obviously, by pasting unilateral steel-slab-bonded can improve the bearing capacity of this kind of floor and to improve its stiffness to meet the requirement of the floor normal's use. Some experiment studies about the concrete beam whose tensile area reinforced by unilateral steel-slab-bonded have shown that ${ }^{[2,3,4]}$, unilateral steel-slab-bonded can improve the flexural bearing capacity and stiffness, reduce the component deformation; The study about by pasting sticking steel in the component pressure zone to improve compressive bearing capacity is less, the literature ${ }^{[5]}$ analyze mechanical characteristics of compressive zone reinforced by pasting sticking steel based on the Internal force superposition principle. In some projects, especially, some projects which have requirement for bearing capacity of slab and the deflection,this text proposed design method of using bilateral steel-slab-bonded to reinforce concrete floor, which improve the bearing capacity of the component cross section pressure areas by the steel plate.Avoiding brittle ultimate failure station controlled by concrete crush, improving the flexural bearing capacity and the section stiffness of the floor slab, reduce the deformation of floor slab. The floor of bilateral steel-slab-bonded are introduced below. According to section internal force equation relation and classical theory about bended component rigidity, the formula of flexural capacity and section rigidity about FC floor strengthened with bilateral steel-slab-bonded are deduced. 


\section{Double side glued steel reinforced concrete slab}

Technology of reinforced concrete floor slab with double side pasting steel plate, that is, at the same position in the concrete floor at the same location at the same time, paste steel plate, the effective anchoring and connection of the upper and lower surfaces of the upper and lower surfaces of the upper and lower surfaces of the upper and lower surfaces by the chemical, improving the compressive bearing capacity of the section of the floor under the steelplate, so as to improve the flexural bearing capacity and section stiffness of the floor slab, as shown in Figure 1. The construction technology of double - sided glued steel reinforced concrete slab is the same as the traditional one - sided sticky steel reinforcement method, but it is necessary to take reliable structural measures to ensure the effective bonding between the upper surface of steel and the floor slab and to prevent the anchoring failure or buckling of the steel plate by the tension and pressure. Should be combined with the construction of the structural drawings and used the steel bar to location the reinforced bars of floor slabs, we need to avoid hurting the floor reinforced bars during drilling.

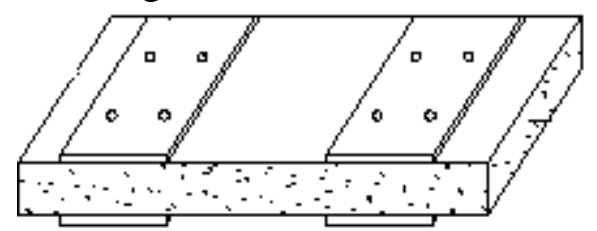

Fig.1 Schematic diagram of the concrete slab strengthened with double side pasting steel plate

\section{Calculation of flexural bearing capacity of reinforcement concrete Slabs Strengthened with double side glued steel}

Failure mode and basic assumption.Similar to reinforced concrete beam with single side sticky steel, double bonded steel reinforced concrete floor slab also exist three kinds of failure mode: Bond failure. Bending failure、Shear failure, it is concerned the floor mainly withstand the uniform load and the failure is main controlled by the bending bearing capacity of floor. The ideal failure mode of double bonded steel reinforced concrete slab is the increase of the external load, the tensile strength of the steel first and the newly added steel plates are second active yield strength. Pressure zone concrete will reach the ultimate compressive strain which is the failure mode of the reinforcement.

When the bearing capacity of concrete slabs strengthened with double side glued steel is calculated, the basic assumption is used: (1) Cooperative work of concrete slab and upper and lower surface of the steel sheet, section strain of member to meet the assumption of plane cross section; (2) The steel plate and concrete are bonded well, ignoring the relative slip between the two; (3) It is generally that the temperature stress reinforced bars or structure distributing bars in the middle and upper part of the floor slab, without considering its effect on the bearing capacity and the short-term stiffness; (4) After the reinforcement, the floor slab it is damaged by the tension steelplate 、 the tension of the reinforced bars yield and Compression zone concrete crushed as a symbol; (5) Without considering the effect of concrete tensile strength, shrinkage, creep and temperature stress.

Normal section bearing capacity calculation.Reinforcement of most floors is in the condition of partial unloading or loading, there is a certain deflection in front of the reinforcement, concrete and reinforced bars have certain stress and strain and at this time, the new steel plate in the load increase before almost no stress and strain, after the load increase, steelplate is produced with stress and strain, therefore, the stress and strain of the new steel plate is lagging behind that of the original strain, belong to the secondary load structure. When the reinforcement floor slab reaches the damage state, steel sheets may not reach their ultimate tensile or compressive bearing capacity, calculation formula of bending bearing capacity of a double band beam can not be used and need to be based on existing deformation and flat section assumption, it is considering the actual stress level of the front floor slab by before reinforcement, calculation formula is derived reasonable. For the floor slabs with partial unloading or loading do double - sided bonding steel plate reinforcement, the internal force analysis is shown in Figure 2. 


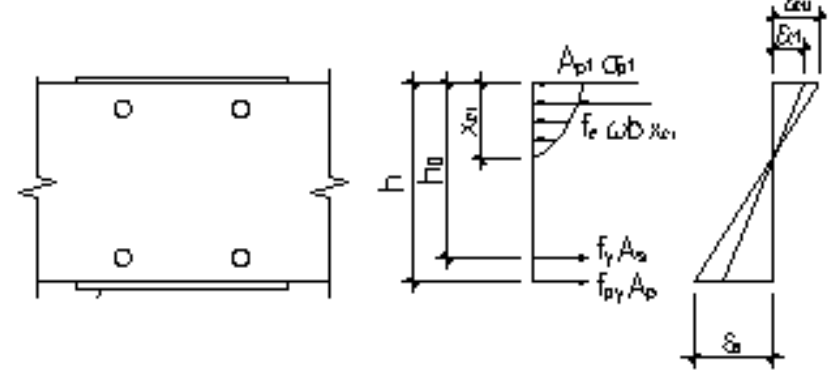

Fig.2 Schematic analysis of section internal force

Pre-reinforcement, concrete compressive strain under the action of vertical load is $\varepsilon_{c 1}$, after reinforcement the structure reaches its limit state concrete compressive strain is $\varepsilon_{c u}=0.0033$, Then concrete strain increment is

$$
\Delta \varepsilon=\varepsilon_{c u}-\varepsilon_{c 1}
$$

Pressure zone plate stress is

$$
\sigma_{p 1}=\Delta \varepsilon \cdot E_{s}
$$

By figure two, according to the section internal force balance relationship:

$$
\begin{gathered}
\alpha_{1} f_{c} b x+\sigma_{p 1} A_{p 1}=f_{y} A_{s}+f_{p y} A_{p} \\
M=\alpha_{1} f_{c} b x\left(h-\frac{x}{2}\right)+\kappa \sigma_{p 1} A_{p 1} h-f_{y} A_{s}^{\prime} a_{s} \\
M=f_{y} A_{s}\left(h_{0}-\frac{x}{2}\right)+\kappa f_{p y} A_{p}\left(h-\frac{x}{2}\right)-\kappa \sigma_{p 1} A_{p 1} \frac{x}{2}
\end{gathered}
$$

Compression zone height from (3) type, Relative boundary pressure zone height according to (6) type, and should meet the requirements of reinforcement design specification

$$
\zeta_{b}=0.8 \zeta_{c b}=\frac{0.8 x_{c}}{h_{0}}=\frac{0.8}{1+f_{y} / 0.0033 E_{s}}
$$

Above (1) (6) type, the floor under surface reinforced steel plate (tension) sectional area is $A_{p}$ and tensile strength design value is $f_{y p}$. The floor surface reinforced steel plate (pressure) cross section area is $A_{p 1}$ and compressive stress is $\sigma_{p 1}$. Rest symbolic meaning design specification for reinforced concrete structures. Using (4) or (5) to calculate the flexural bearing capacity, the practical effect of the joint work of the steel, the structure and the concrete is considered. The appropriate reduction of the bearing capacity of the steel plate, the value of the reduction factor is between 0.95 and 0.85 . Calculation formula for stress of plate under compression (2), the stress level of the steel plate is determined by the strain of the concrete slab in the actual stress state. When $\varepsilon_{c 1}=0$, it is completely unloaded. At this time, the new steel plate and concrete deformation are to reach agreement, it can be regarded as a force situation; When $\varepsilon_{c 1}=\varepsilon_{c u}=$ 0.0033 , The pressure zone concrete has reached the limit strain state.Additional compressive stress due to the increase of the load will be entirely from the pressure area of the adhesive plate, In this case, the concrete may be crushed at any moment. It is not allowed to appear in the design. The steel reinforcement for most of the floors, the concrete strain state in the pressure zone is between the two. 


\section{Rigidity calculation of floor slab with double side glued steel}

Early in the application of reinforced concrete structures, The design and calculation of the bearing capacity of the members are all reference to the calculation method of the homogeneous elastic materials, The main principle is to put the steel bar on the cross section, By the ratio of elastic modulus to the fold change and get equivalent material conversion section, establish the corresponding calculation formula, That is the effective moment of inertia ${ }^{[7]}$. This principle and method are still in the design and analysis of concrete structures, Such as Prestressed reinforced concrete construction pre-cracked, stiffness analysis, fatigue calculation, etc. Stiffness analysis method is based on the curvature formula of components, geometrical conditions, constitutive relation of materials and the equation of section mechanics, the formula for calculating the section stiffness of the component is the use of the stiffness analysis method and the ${ }^{[8]}$.

Based on classical effective inertia moment method and stiffness analysis method, a formula for calculating the stiffness of the double sided adhesive steel plate is derived and established, the equivalent internal force distribution and the average strain of the member section are shown in figure three. For ease of engineering application, some assumptions and simplified calculation are carried out in the formula:(1) The steel plate was converted to concrete by the ratio of elastic modulus of steel plate; (2) Considering two factors such as force and adhesion, It will be affected by the pull of the plate by the reduction factor of 0.8 and then incorporated into the reinforced part; (3) In calculating floor deflection, considering the effect of the load on the deflection of the long term, the influence coefficient $\theta$ is 2.0 .
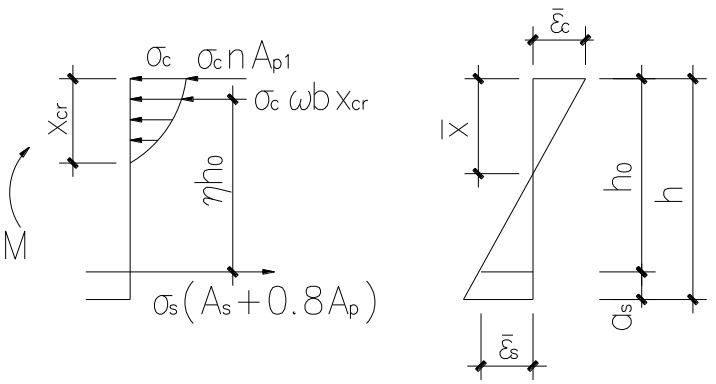

Fig.3 Equivalent internal force distribution and average strain diagram of the member section Member section deformation condition.From the previous hypothesis, the average strain of the floor slab in normal use stage is still in line with the assumption of plane section, the average curvature of the cross section can be expressed as:

$$
\frac{1}{\rho}=\frac{\overline{\varepsilon_{c}}+\overline{\varepsilon_{s}}}{h}
$$

Formula: Average pressure strain for concrete is $\overline{\varepsilon_{c}}$, taking as $\overline{\varepsilon_{c}}=\varepsilon_{c}$;Average compressive strain of steel bar is $\overline{\varepsilon_{s}}$, taking as $\overline{\varepsilon_{s}}=\psi_{s} \varepsilon_{s}$, Uneven coefficient of tension between the crack and the tensile steel plate is $\psi_{s}, \psi_{s}=1.1-\frac{0.65 f_{t k}}{\rho_{t e}\left(\sigma_{s k 1}+\sigma_{s k 2}\right)}$, Stress value of tension bar in the front and back of the glued steel are $\sigma_{s k 1}$ and $\sigma_{s k 2}$.

Constitutive relation of materials.In normal use stage, the relationship between stress and strain of concrete and steel bar:

$$
\sigma_{c}=\varepsilon_{c} \lambda E_{0} \approx \overline{\varepsilon_{c}} \lambda E_{0}
$$

$$
\sigma_{s}=\varepsilon_{s} E_{s}=\frac{\overline{\varepsilon_{s}}}{\psi_{s}} E_{s}
$$

By (8), (9) - type transformation:

$$
\overline{\varepsilon_{c}}=\frac{\sigma_{c}}{\lambda E_{0}}
$$

$$
\overline{\varepsilon_{s}}=\frac{\psi_{s} \sigma_{s}}{E_{s}}
$$


Formula: Compression deformation coefficient of concrete is $\lambda$, elastic modulus of concrete and reinforced concrete (steel plate) are $E_{0}$ and $E_{s}$, in the simple, the elastic modulus of the reinforced and reinforced steel plates is equal to that of the steel plates.

Equilibrium equation.By the assumption that the stress of the concrete in the section of the section is neglected, the moment of the joint forces of the reinforced concrete and the compression zone is respectively. When the pull of the reinforcing bar is removed, it will be incorporated into the tension of the steel plate due to the reduction of the tensile strength, so the equation does not appear to be influenced by the pull of the steel plate to the bending moment of the steel bar.

$$
M=\sigma_{c}\left(n A_{p 1} h+\omega b x_{c r} \eta h_{0}\right) \quad(12) \quad M=\left(A_{s}+0.8 A_{p}\right) \sigma_{s} \eta h_{0}
$$

From (12), (13) where conversion can be obtained:

$$
\sigma_{c}=\frac{M}{\left(n A_{p 1} h+\omega \eta x_{c r} b h_{0}\right)} \quad \text { (14) } \quad \sigma_{s}=\frac{M}{\eta\left(A_{s}+0.8 A_{p}\right) h_{0}}
$$

In the formula, $\omega$ is the stress factor graphics complete nip; $\eta$ is the cross section of the arm fracture coefficient.

The (10), (11), (14), (15) turn into (7), and remember $n=E_{s} / E_{0}, \mu=\left(A_{s}+0.8 A_{p}\right) / b h_{0}$, then an average curvature:

$$
\frac{1}{\rho}=\frac{M}{E_{s}\left(A_{s}+0.8 A_{p}\right) h_{0}^{2}}\left[\frac{\psi_{s}}{\eta}+\frac{n \mu}{\lambda\left(n m / h_{0}+\omega \eta x_{c r} / h_{0}\right)}\right]
$$

Therefore, the average secant stiffness floor section as follows:

$$
B=\frac{M}{1 / \rho}=\frac{E_{s}\left(A_{s}+0.8 A_{p}\right) h_{0}^{2}}{\frac{\psi_{s}}{\eta}+\frac{n \mu}{\lambda\left(n m / h_{0}+\omega \eta x_{c r} / h_{0}\right)}}
$$

In the formula, and so $E_{s} A_{s} A_{p} h_{0}$ and $n=E_{s} / E_{0}$ and $\mu$ are determined value, $\psi_{s}$ can be calculated ${ }^{[2]}$ According to the formula, and other remaining coefficients $\eta, \lambda$ and $\omega$ values are changed with the bending moment, according to the experimental results must be analyzed, $\eta$ crack in which the arm section desirability factor $\eta=0.87 . K=\frac{n \mu}{\lambda\left(n m / h_{0}+\omega \eta x_{c r} / h_{0}\right)}$ Comprehensive reflect changes after pasting steel floor nip section stiffness, known as double-sided sticky steel reinforcement concrete slabs comprehensive section stiffness coefficient of variation, refer nip dual reinforced concrete beam reinforcement force test results ${ }^{[7]}$, a cross-sectional $K$ comprehensive stiffness coefficient of variation is desirable

$$
K=\frac{n \mu}{\lambda\left(n m / h_{0}+\omega \eta x_{c r} / h_{0}\right)}=0.2+6 n \mu /\left(1+3.5 n A_{p 1} / b h_{0}\right)
$$

The simplified formula (18) and $\eta$ values are substituted into the formula (17), that was the double-sided sticky steel reinforced concrete slab stiffness sectional average formula:

$$
B=\frac{E_{s}\left(A_{s}+0.8 A_{p}\right) h_{0}^{2}}{1.15 \psi_{s}+0.2+6 n \mu\left(1+3.5 n A_{p 1} / b h_{0}\right)}
$$

For the moment mainly exposed concrete slabs, the inner surface of the shaft and the outer surface of the shear force vertical load generated by the use of phase distortion negligible impact on, while the largest cross-section according to the moment in the sector at the moment with the number of the minimum stiffness calculated by the formula (19) returns a sectional floor after short-term stiffness can be calculated according to the standard combination of load effects and consider the long-term effect of the impact of long-term load stiffness, deflection across the floor and then obtained based on the principle of virtual work. 


\section{Structural measures}

To ensure that slab sided paste steel and concrete can effectively work together, in addition to structural measures prescribed norms bonded steel reinforcement law must be taken, we propose the following two structural measures in this paper, shown in Figure 4.

(1)Short transverse reinforcement welded steel surface, the floor surface of the corresponding parts of the pre-grooved to improve the mechanical bite force between the plate and the floor, increasing between the two anti-slip capability.

(2)Reference member surface anchoring mechanism embedded hard reinforcement methods vertical short bars welded steel surface, which is embedded inside the short reinforced concrete, strengthening the bond anchoring effect of steel and concrete floor.
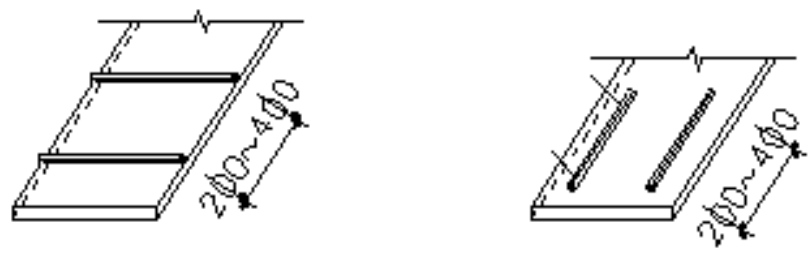

Fig.4 Schematic diagram of structural measures

\section{Summary}

Paste sided steel reinforced concrete floor slab method can greatly improve the use of flexural capacity and rigidity, reduce floor deflection and cracks after the load increases. According sided sticky steel reinforced concrete beams related experimental research and practical floor reinforcement needs, first proposed the bilateral steel reinforced concrete slab technology, combined with a cross-sectional internal force balance relations and solving the classical theory of flexural rigidity of members, and to consider strengthening the process the situation is not completely uninstalled floor, deduced and established a double-sided sticky floor steel reinforcement bending capacity and rigidity formulas, with "reinforced concrete structure design specifications" used in conjunction, can better meet the requirements for floor deflection have reinforcement engineering. Steel Reinforcement for double-sided sticky stringent performance requirements for bonding between steel and slab, we proposed to two structural measures of improving the bonding effect between the steel plates and slabs, these structural measures apply equally to single bonded steel reinforced concrete beams and other flexural members.

\section{Reference}

[1] Reinforced concrete structure design code (GB50367-2006) Beijing: China Building Industry Press, 2006.

[2] Li Wensheng, Liu Zhean ,Stick steel reinforcement beam under short-term load stiffness and deflection [J],Reinforced building structure, 2004.3: 16-18.

[3] Wang Tianwen, Concrete member stick steel reinforcement calculation problems of normal section[J],Journal of the building structure, 1997.11: 12-14.

[4] Zhao Chonghua, Wang Tianwen, The deflection of concrete beams glued steel structure after checking [J],Building Technology Development, 2002.9: 12-15.

[5] Zhou Junwen, Xu Jiaen, Yang Yong, Zhang Weiming,Concrete Beams nip sticky steel reinforcement capacity analysis [J] ,Hubei Institute of Technology, 2002.3: 34-36.

[6] Wan Molin, Han Jiyun,Concrete structure reinforcement technology [M],Beijing: China Building Industry Press, 1991. 
[7] Guo Zhenhai, Shi Xudong,Reinforced concrete principles and analysis [M] ,Beijing: Tsinghua University Press, 2003: 263-267.

[8] Design of Concrete Structures (GB50010-2002) [S],Beijing: China Building Industry Press, 2002. 